\title{
Impact of Physiological Neutral pH on Morphology of Francisella tularensis - An Electron Microscopic Study
}

\author{
Catherine M. Wastella ${ }^{1,2}$, David R. Williamson ${ }^{3}$, Girish S. Kirimaneswara ${ }^{3}$ and Gang Ning ${ }^{1,2}$ \\ ${ }^{1 .}$ Microscopy Facility, The Huck Institutes of the Life Sciences and ${ }^{2 .}$ Department of Biology, \\ Pennsylvania State University, University Park, Pennsylvania, USA. ${ }^{3 \cdot}$ Department of Veterinary and \\ Biomedical Sciences, Pennsylvania State University, University Park, Pennsylvania, USA.
}

Francisella tularensis is an infectious, gram-negative, coccobacilli bacterium that is responsible for causing the disease Tularemia, via varying routes in both animal and human. F. tularensis is a facultative intracellular bacterium which grows in the cytosol of host cells and in media of $\mathrm{pH} \sim 5.5$ to 7.5. The overall physiological neutral $\mathrm{pH}$ of most niches of the human body is about 7.4, therefore, any effective pathogen must be able to not only survive but also proliferate at this slightly alkaline $\mathrm{pH}$, especially in many intracellular conditions. Our study demonstrates that $F$. tularensis subsp holarctica grows at a reduced rate at $\mathrm{pH} 7.4$ compared to $\mathrm{pH6.4}$, suggesting that $\mathrm{pH} 7.4$ presents a challenge to which the bacterium must adapt. Although ultrastructure of $F$. tularensis in acidic endosomes and lysosomes as well as in neutral $\mathrm{pH}$ of cytosol, particularly in macrophages, has been reported, the impact of neutral $\mathrm{pH}$ environment on morphology of the bacteria has not been reported. In this study, we used different EM techniques to reveal morphological differences between $F$. tularensis grown in media at pH6.4 and 7.4.

Francisella tularensis subsp. holarctica Live Vaccine Strain (LVS) was obtained from Albany Medical College, NY. Liquid cultures of $F$. tularensis were routinely grown in modified Brain Heart Infusion Broth, Modified (BD BBL \#299070) at $37^{\circ} \mathrm{C}$ in an orbital shaker operating at 200rpm. Mid-log phase cells grown in pH6.4 and pH7.4 were harvested at a density of $2 \times 10^{9} / \mathrm{mL}$ for microscopic study with (1) negative staining, (2) conventional TEM, (3) cryo-fixation and freeze-substitution TEM, and (4) SEM. Cells were negatively stained with $2 \%$ phosphotungstic acid (PTA) [1]. For conventional TEM, cells were fixed in 2\% glutaraldehyde (GA) and embedded in Epoxy resin; thin sections were cut and observed under a TEM at $80 \mathrm{kV}$. Cryo-fixation was performed by plug-freezing cells into LN2-cooled liquid propane at about $-160^{\circ} \mathrm{C}$ using a Leica $\mathrm{CPC}$; the frozen samples were processed according rapid freeze-substitution protocol by McDonald and Webb [2] with modifications. Cells were also fixed with $2 \%$ GA and placed on glass coverslip coated with poly-L-lysine; cells were then critical-point dried and sputter coated with Iridium for SEM observation at $2 \mathrm{kV}$.

Overall subtle changes were detected in cells grown in media of pH7.4 vs. pH6.4 upon careful observations. TEM images of negative-stained bacteria revealed that bacteria grown at $\mathrm{pH} 7.4$ appeared to have a transparent cell wall and occasional disruptions of the membrane when compared to the membrane of bacteria grown at pH6.4 cells (Figure 1). Images of thin-sectioned samples of conventional and cryo-prepared samples did not show significant differences in thickness and architectural alteration between both groups while a slight tendency of separation of outer and inner membrane was noticed in cells grown at pH7.4 (Figure 2). The images taken with SEM showcased the shape and topological differences between the bacteria at pH6.4 and 7.4. At pH6.4, cells maintained their shape of bacillus or coccus, and looked overall better and healthier than at pH7.4. Cells grown at $\mathrm{pH} 7.4$ lost their rounded edges, exhibited surface dimpling, and no longer conformed to prototypical coccobacillus morphology 
(Figure 3). The present study demonstrated that the physiological neutral $\mathrm{pH}$ has an impact on morphology and cell growth of $F$. tularensis.

\section{References:}

[1] Ark NM and Mann JB, Microb Pathog 51 (2011), p. 110.

[2] McDonald KL and Webb RI, J Micros 234 (2011), p. 227.

[4] This study is partially supported by NIH AI077917.
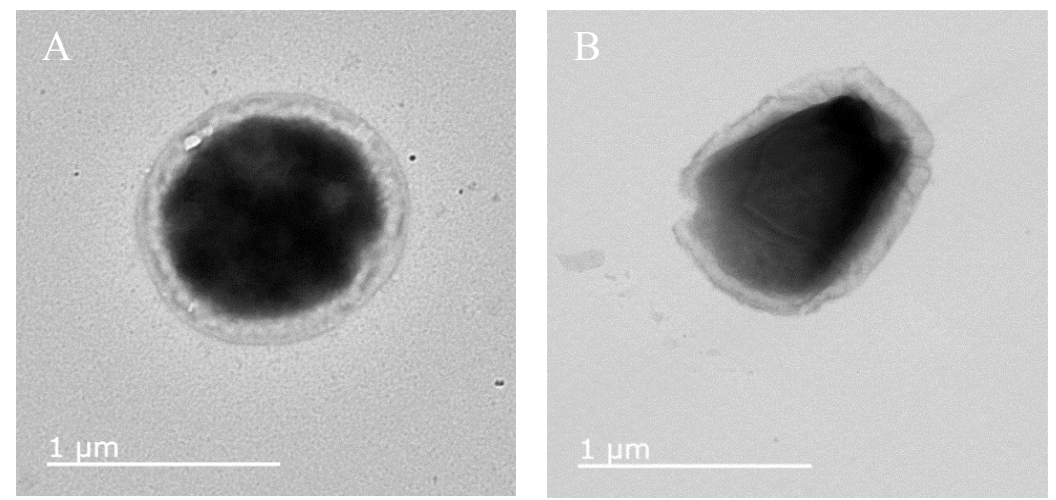

Figure 1. TEM images of negative-stained F. tularensis from pH6.4 (A) and 7.4 (B). The cell wall from pH7.4 appears more transparent and have uneven thickness.
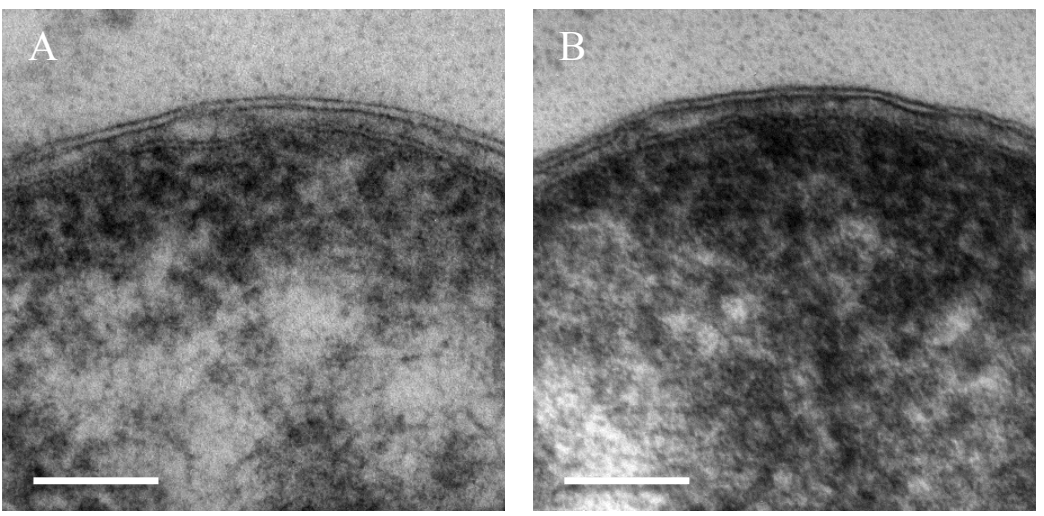

Figure 2. TEM images of thin-sectioned $F$. tularensis from pH6.4 (A) and 7.4 (B). There are no outright visible differences in the cell wall and membranes. Scale bars $=100 \mathrm{~nm}$.
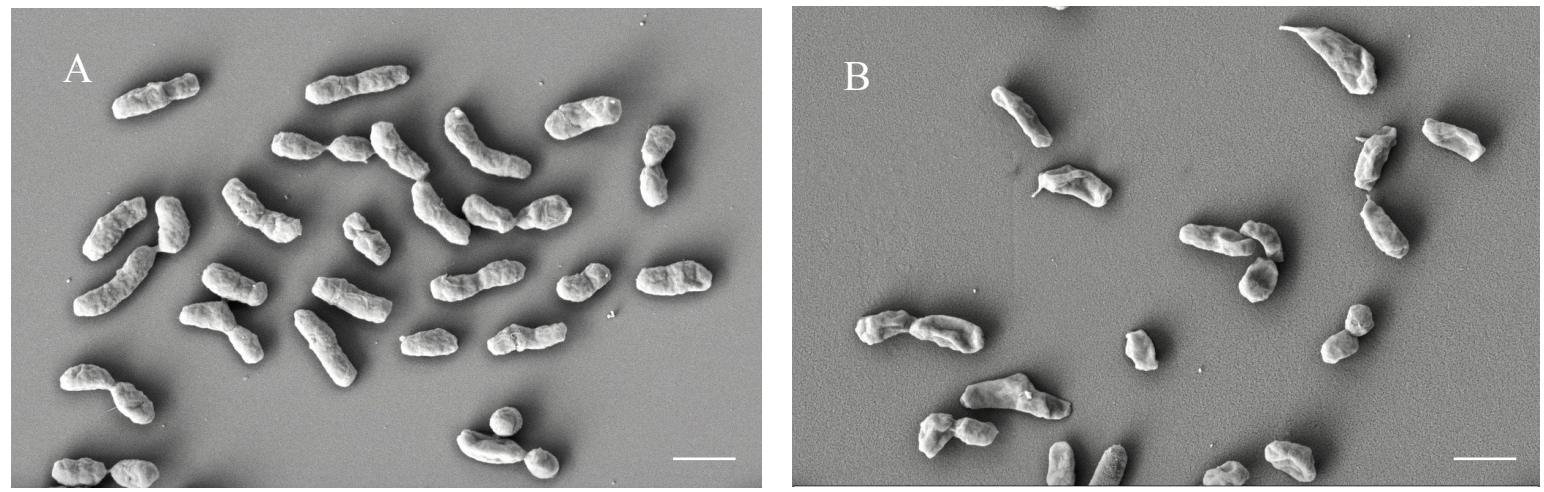

Figure 3. SEM images of $F$. tularensis from pH6.4 (A) and $7.4(\mathrm{~B})$. The cells from $\mathrm{pH} 7.4$ appear flattened and collapsed, and some have protrusions. Scale bars $=1 \mu \mathrm{m}$. 